\title{
FATORES QUE AUXILIAM NA LOCALIZAÇÃO DE CENTRO DE DISTRIBUIÇÃO: UMA ANÁLISE BIBLIOMÉTRICA
}

\section{FACTORS THAT ASSIST IN THE LOCATION OF A DISTRIBUTION CENTER: A BIBLIOMETRIC} ANALYSIS

\author{
Rodolfo Santos Bertolo ${ }^{*}$, \& Rodrigo Randow de Freitas ${ }^{2}$ \\ ${ }^{12}$ Centro Universitário Norte do Espírito Santo da Universidade Federal do Espírito Santo. \\ 1*rodolfobertolo51@gmail.com ${ }^{2}$ rodrigo.r.freitas@ufes.br
}

\section{ARTIGO INFO.}

Recebido em: 07.11.2020

Aprovado em: 17.12.2020

Disponibilizado em: 31.03.2021

\section{Palavras-chave:}

Centro de Distribuição; Logística; Bibliometria; Localização.

\section{KEYWORDS:}

Distribution center; Logistics; Bibliometric; Location.

*Autor Correspondente: Bertolo, R. S.

\section{RESUMO}

Centros de Distribuição (CD) são instalações fundamentais para a Cadeia de Suprimentos e são estruturados de acordo com a sua demanda e localização geográfica, para poder atender seus compradores. Quando estão estrategicamente localizados, os centros de distribuição de uma empresa, proporcionam redução significativa dos custos logísticos. Assim, este estudo tem como objetivo mapear a atividade científica (bibliometria) com base no tema: fatores que auxiliam na localização de um CD, utilizando a princípio todo o período de abrangência das principais bases de dados disponíveis: Web of Science - WOS, Scopus e Scielo. O processo selecionou 11 artigos relevantes, alinhados ao tema, realizou-se ainda uma análise bibliométrica qualitativa do portfólio a fim de caracterizar e analisar os artigos. A maioria dos trabalhos trabalhou com a teoria Fuzzy e outros métodos de análise multicritérios. E pode-se observar que acesso, custo, impacto ambiental e recursos são mencionados em vários artigos do portfólio final analisado.

\begin{abstract}
Distribution Centers (DC) are fundamental facilities for the Supply Chain and are structured according to their demand and geographic location, in order to serve their buyers. When strategically located, a company's distribution centers provide a significant reduction in logistics costs. Thus, this study aims to map the scientific activity (bibliometrics) based on the theme: factors that help in the location of a $C D$, using in principle the entire coverage period of the main available databases: Web of Science - WOS, Scopus and Scielo. The process selected 11 relevant articles, aligned with the theme, a qualitative bibliometric analysis of the portfolio was carried out in order to characterize and analyze the articles. Most works have worked with Fuzzy theory and other methods of multicriteria analysis. And it can be seen that access, cost, environmental impact and resources are mentioned in several articles in the final analyzed portfolio.
\end{abstract}




\section{INTRODUÇÃO}

O mercado globalizado exige diversas modificações com muita rapidez por parte das organizações, para que estas se mantem inseridas neste mercado. Com isso, verifica-se $p$ aumento do número de indivíduos que se interessam na área de logística, podendo ser a logística de transportes ou a logística de organizações. Sendo que o objetivo primordial vem a ser aperfeiçoamento, agilidade, aumento da produção, qualidade dos produtos e serviços e, a permanência no mercado.

A logística pode ser definida como a integração da administração de materiais com a distribuição física (Ballou, 1993). A logística, como lembra Ballou (2001) "é um processo de planejamento, implementação e controle de fluxo desde o ponto de origem até a disponibilização ao consumidor”.

De acordo com números divulgados por portais especializados em logística, como ILOS (2017), e por reportagens de jornais credenciados, como Samor (2018), as operações logísticas representaram para o Brasil em 2017 um custo de aproximadamente 820 bilhões de reais. Previa-se para 2020 um menor custo com logística, visto que o orçamento destinado para infraestrutura de transporte seria o menor dos últimos 16 anos, aumentando os custos das empresas com transporte e reduzindo a competitividade. Além da indicação de recuração da economia com a previsão de crescimento do PID em mais de 2\% (E-commerce Brasil, 2019).

Porém, devido à pandemia, a logística também foi comprometida. A NTC\&Logística apurou uma queda de 26,14\% no volume de cargas em relação à operação normal das transportadoras. Para carga fracionadas, a queda chegou a $29,81 \%$, enquanto para cargas de lotação o declínio apresentado foi de 22,91\% (Pelúcio, 2020), o que impacta diretamente nos custos de transportes logísticos.

Sendo que, de 1 a 2/3 dos custos logísticos são originados pelos custos de transporte, por meio das despesas relacionadas aos fretes cobrados, para deslocamento dos pontos de produção aos pontos de consumo. Assim, justifica-se uma análise criteriosa da localização dos centros de distribuição e, até mesmo, dos centros de produção de uma empresa (Ballou, 2001).

Centros de Distribuição (CD) são instalações fundamentais para a Cadeia de Suprimentos, pois têm por objetivo providenciar entregas mais rápidas de produtos aos clientes, equilibrar o estoque com a demanda do mercado e melhorar a comunicação de atendimento ao cliente. (Harper, 2010).

Os CD são estruturados de acordo com a sua demanda e localização geográfica, para assim poder atender seus compradores. Destaca-se, inicialmente, que a localização ideal de um centro de distribuição, seja qual for o segmento do negócio, é justificada principalmente pela redução dos custos operacionais, envolvendo diretamente os custos de transporte (Ramos, 2015).

Outras ações consideradas importantes no contexto de logística de distribuição são: roteirização dos veículos de forma otimizada, minimização de distâncias percorridas nas rotas de entrega e a ocupação maximizada da frota (Ramos, 2015).

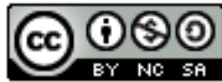


O aumento das atividades de distribuição urbana no Brasil e a ampliação das políticas de restrição de circulação urbana, geraram novos desafios para as empresas de serviços logísticos e de transportes do país (ILOS, 2014).

Com isso, tem-se que os centros de distribuição localizados mais próximos dos centros de consumo, permitem a implantação de veículos com menores capacidades de carga, que podem proporcionar menos congestionamentos, maiores velocidades de deslocamento e baixo desgaste da estrutura viária dos grandes centros urbanos. Quando estão estrategicamente localizados, os centros de distribuição de uma empresa, proporcionam redução significativa dos custos logísticos (Ramos, 2015).

Com a implantação dos CDs, é possível notar mudanças no ambiente da cidade. As vantagens são: entregas mais rápidas, redução de impacto no trânsito, respeito à legislação de tráfego pelos veículos, redução da poluição atmosférica, fornecimento organizado de suprimentos, etc (Carvalho et al., 2019).

A relevância da logística de distribuição e sua influência no país, justifica a importância de se realizar um estudo bibliométrico acerca do assunto, principalmente pela relação dos custos logísticos e a localização dos centros de distribuição. A análise bibliométrica auxilia no entendimento de como o conhecimento científico é difundido e incorporado entre os autores e a comunidade acadêmica em geral (Noronha et al., 2002; Cunha et al., 2018). A bibliometria vem se tornando uma ferramenta indispensável na avaliação da produção de instituições, áreas, temas e pesquisadores.

De acordo com Chueke e Amatucci (2015), um conceito ao qual o estudo bibliométrico se apoia, é a premissa de que o conhecimento científico é cumulativo e cooperativo, e é construído a partir de resultados de pesquisas que endereçam lacunas a serem investigadas no futuro. Desta forma, os autores concluem que os artigos que buscam sistematizar a produção científica contribuem para o incremento da relevância e rigor de novas pesquisas, e podem servir como base para estudos comparados.

Os estudos que têm como base a análise bibliométrica vem recebendo destaque no âmbito acadêmico, visto que esta seja reconhecida pela sua relevância para quantificar, classificar e avaliar trabalhos científicos (Gomes \& Freitas, 2019). Dessa forma, este trabalho identifica os fatores que auxiliam na localização de um $\mathrm{CD}$, utilizando ferramentas de pesquisa acadêmica. O objetivo é mapear a atividade científica com base no tema, utilizando a princípio todo o período de abrangência das principais bases de dados disponíveis: Web of Science - WOS, Scopus e Scielo. Assim, essa pesquisa ela utiliza a ferramenta de pesquisa acadêmica, como foi citado acima, como base no tema "Fatores que auxiliam na localização de centro de distribuição: uma análise bibliométrica" fim de obter um melhor entendimento acerca de conceitos, estruturas e aplicações de trabalhos científicos já produzidos.

\section{Metodologia}

Com a finalidade de estudar e analisar a produção científica, identificando tendências e autores, foi dado início à análise bibliométrica, quantitativa e qualitativa. Com isso, este trabalho utilizou como bases de dados as plataformas Web Of Science - WOS, Scopus e Scielo, nelas 
encontram-se periódicos de grande relevância para o meio acadêmico e científico, possibilitando uma grande cobertura de publicações.

Foram selecionados inicialmente todo o período disponível pelas plataformas, para a WOS temse o período de 1945 até 2020, para a Scopus tem-se o período de 1960 até 2020 e para a Scielo tem-se o período de 1921 até 2020.

Após a definição das bases de dados, foram determinadas as palavras-chave (tags) que determinam o enfoque da pesquisa por meio de tópicos. As palavras-chave escolhidas foram: logística e "centro de distribuição", e suas respectivas traduções no idioma inglês: logistic e "distribution center".

A partir das tags iniciou-se a seleção dos materiais para a composição do portfólio de publicações que serão analisados qualitativamente neste estudo. Primeiramente foram selecionados apenas os documentos do tipo artigo em cada uma das plataformas. Os materiais resultantes desta pesquisa foram exportados para o Microsoft Excel, em forma de planilha de dados para que fosse dada sequência à seleção.

Um novo refino foi realizado, considerando o ano de publicação dos artigos publicados, em que foram selecionados os artigos publicados nos últimos 10 anos.

Uma nova seleção foi realizada, agora com base em um número de corte para os artigos que foram mais citados, conforme o método de Lacerda et al. (2012), dessa forma, os artigos foram ordenados de forma decrescente em relação ao número de citações, sendo selecionados os artigos em que as citações somavam aproximadamente $85 \%$ do total obtido.

A partir da busca pela tag no idioma inglês, os artigos até o momento selecionados nas bases de dados WOS e Scopus ainda se encontravam em grande quantidade para a análise, logo foi necessário aplicar mais um filtro, que foi definir o número amostral considerado válido para análise. Como mencionado por Cay \& Uyan (2013), o tamanho da amostra (número de artigos necessários) depende do tamanho da população (total de artigos) e o grau de confiabilidade desejada para os resultados obtidos.

Para este estudo o tamanho da amostra foi estabelecida pela expressão apresentada na Equação 1 (Dupont \& Plummer, 1990), este cálculo faz-se necessário para garantir a representatividade dos dados coletados e a legitimidade da pesquisa:

$$
n=\frac{Z^{2} \cdot\left(\frac{x}{n}\right) \cdot\left[1-\left(\frac{x}{n}\right)\right] \cdot N}{(N-1) \cdot e^{2}+Z^{2} \cdot\left(\frac{x}{n}\right) \cdot\left[1-\left(\frac{x}{n}\right)\right]}
$$

Com isso, considerando o nível de confiabilidade de 90\%, valor crítico de 1,645 foi possível filtrar os artigos. Por fim, foi realizada a leitura dos títulos e resumos dos artigos das 3 bases de dados nos 2 idiomas, para que fossem selecionados para o portfólio apenas os artigos que condizem com o foco da pesquisa, assim o portfólio de referencial teórico foi composto a partir desta análise quantitativa.

Sendo realizada ainda, a análise qualitativa do portfólio, apresentando uma síntese a respeito das conclusões e perspectivas dos autores destes artigos sobre o tema. A Figura 1 mostra, em termos de etapas, o desdobramento da pesquisa. 


$$
\text { - } 133 \text { - }
$$

Citação (APA): Bertolo, R. S., \& Freitas, R. R. (2021). Fatores que auxiliam na localização de Centro de Distribuição: Uma Análise Bibliométrica. Brazilian Journal of Production Engineering, 7(1), 129-141.

Figura 1. Fluxograma do processo. Elaborado pelo autor.

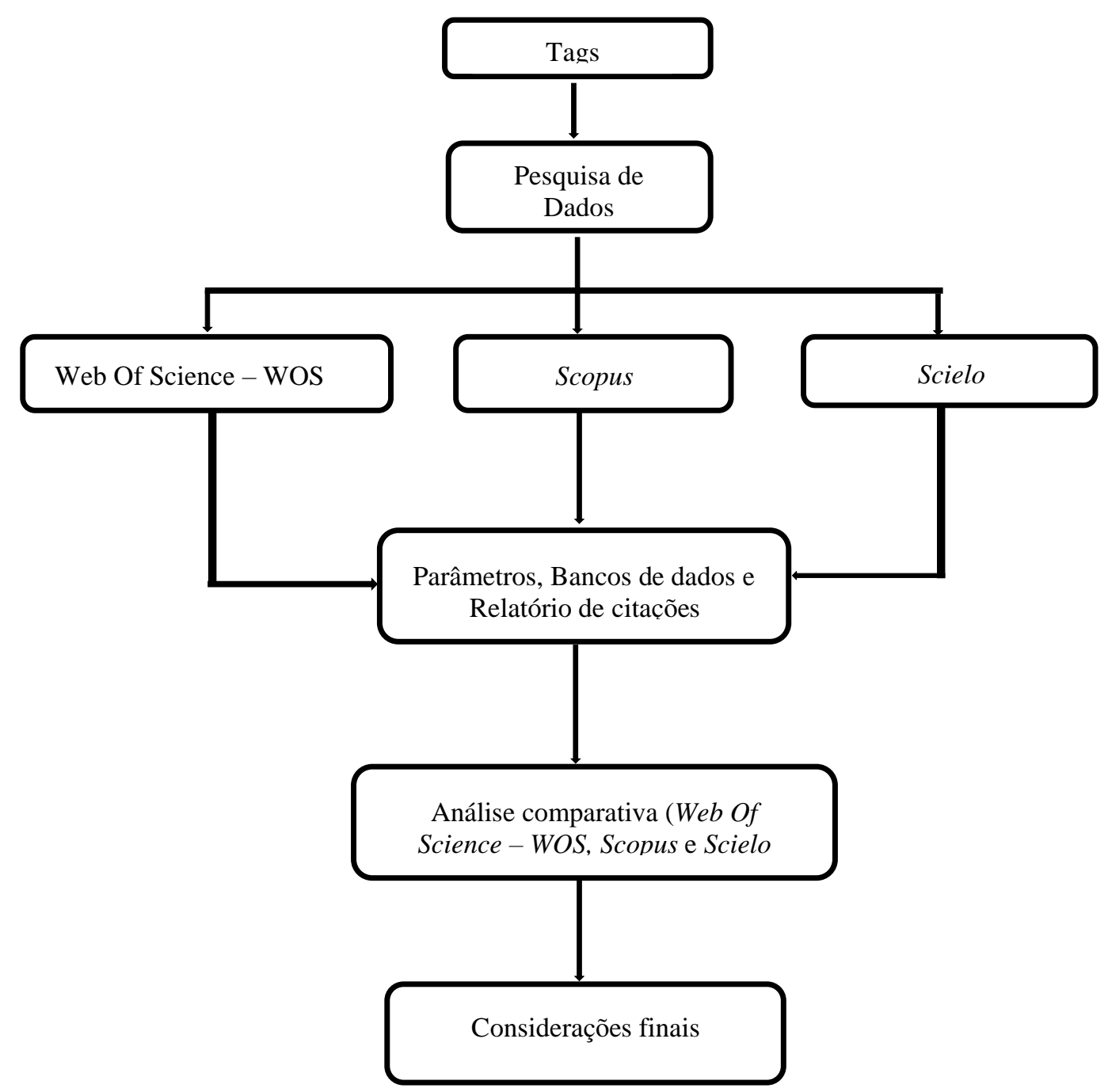

\section{Resultados E DiscusSÃo}

Ao utilizar as palavras-chave mencionadas, e considerando o tempo máximo de publicação de cada uma das bases de dados, foram encontradas a seguinte quantidade e publicações, como mostra a Tabela 1:

Tabela 1. Número de publicações de acordo com a base de dados.

\begin{tabular}{ccc}
\hline Base de dados 29/06/2020 & Inglês & Português \\
\hline Web of Science & 880 & 4 \\
Scopus & 6712 & 23 \\
Scielo & 5 & 4 \\
\hline
\end{tabular}

Fonte: Autores.

Verificou-se que para os idiomas inglês e português a base de dados Scopus foi a que apresentou o maior número de publicações, seguida da WOS e Scielo. A base Scopus é uma das melhores alternativas de base de dados multidisciplinar da literatura científica, contém 20.500 revisores de 5.000 editores e de ampla cobertura de dados de citação (Boyle \& Sherman, 2006).

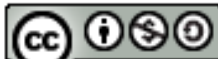




\section{- 134 -}

Citação (APA): Bertolo, R. S., \& Freitas, R. R. (2021). Fatores que auxiliam na localização de Centro de Distribuição: Uma Análise Bibliométrica. Brazilian Journal of Production Engineering, 7(1), 129-141.

Com a seleção dos documentos do tipo artigo, pode-se encontrar a seguinte quantidade de artigos (Tabela 2).

Tabela 2. Número de documentos do tipo artigo de acordo com a base de dados

\begin{tabular}{ccc}
\hline Tipo Artigo & Inglês & Português \\
\hline Web of Science & 352 & 4 \\
Scopus & 4245 & 17 \\
Scielo & 5 & 4 \\
\hline
\end{tabular}

Fonte: Autores.

Sendo que os primeiros artigos de cada uma das bases foram:

- WOS - Chow, G. (1984). Microcomputers in Logistics. Logistics and Transportation Review, 20(4), 471-501.

- Scopus - Shannon, R. E., e Ignizio, J. P. (1970). A heuristic programming algorithm for warehouse location. AIIE Transactions, 2(4), 334-339.

- Scielo - Morales, S. R., Morabito, R., e Widmer, J. A. (1997). Otimização do carregamento de produtos paletizados em caminhões. Gestão \& Produção, 4(2), 234252.

Os Gráficos 1, 2 e 3 mostram a evolução das publicações dos artigos deste tema ao longo dos anos para as três bases de dados pesquisadas.

Gráfico 1. Evolução das publicações WOS

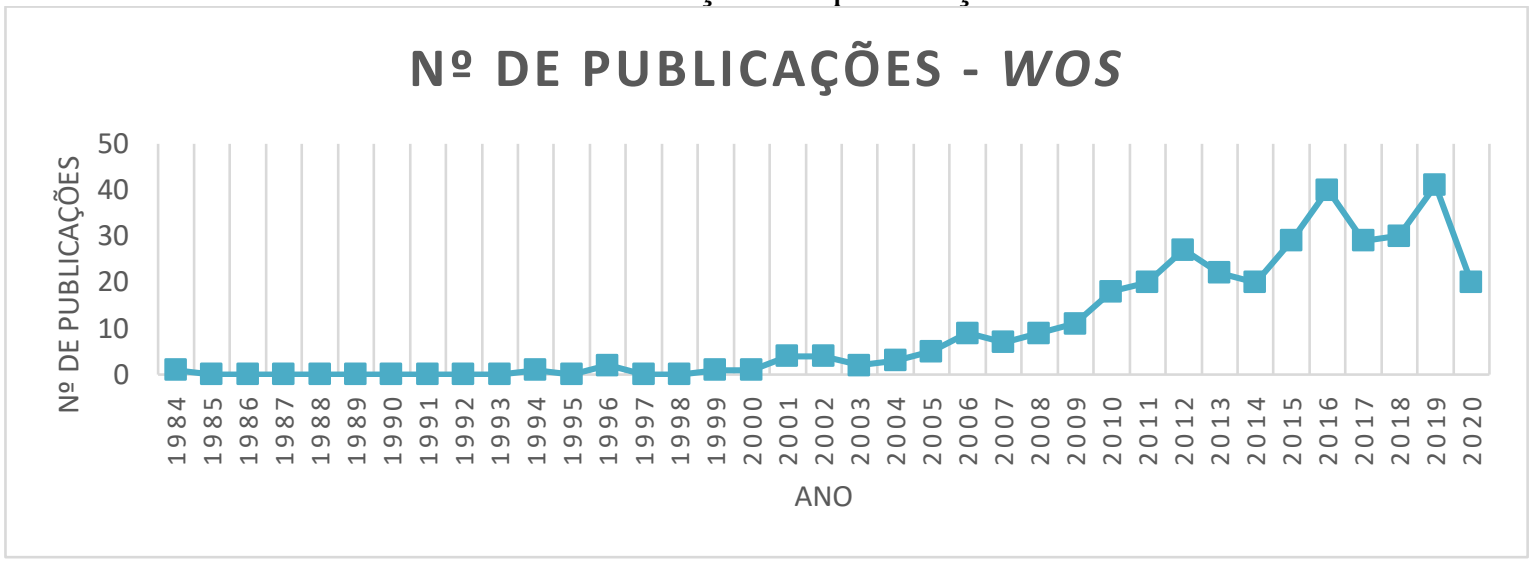

Fonte: Autores.

Gráfico 2. Evolução das publicações Scopus

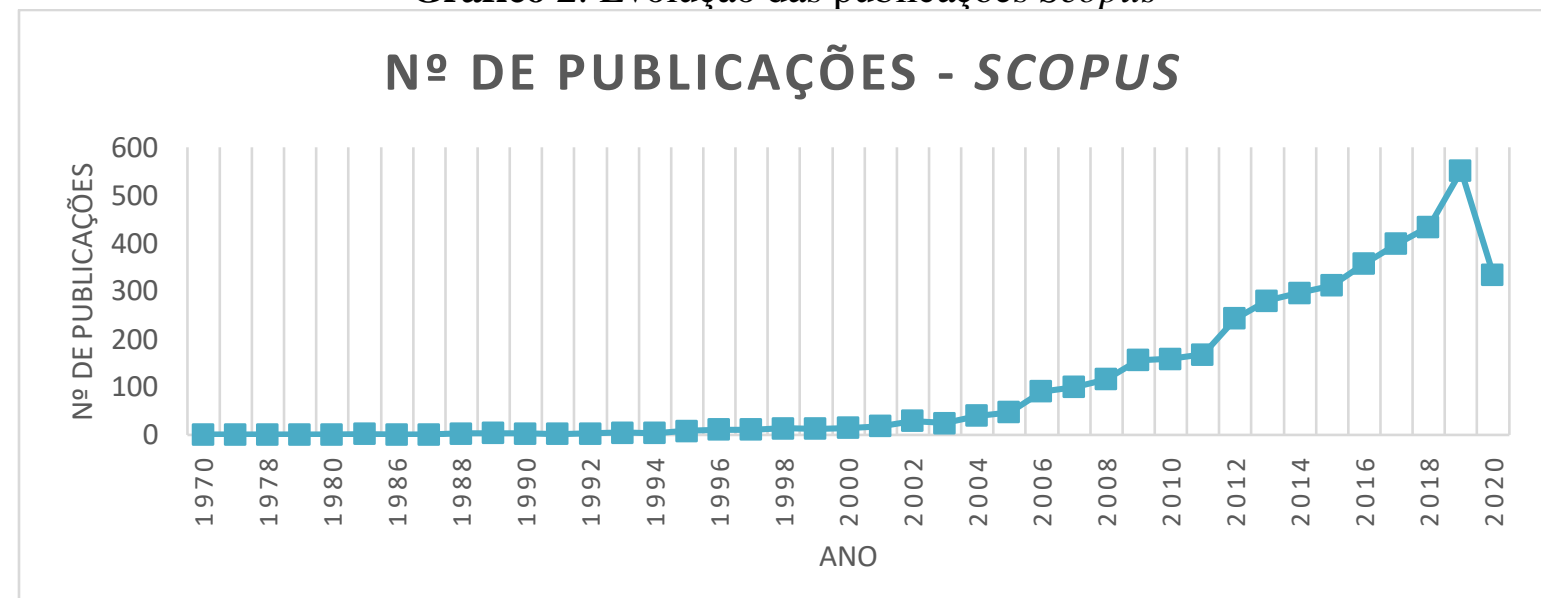

Fonte: Autores. 
Gráfico 3. Evolução das publicações Scielo

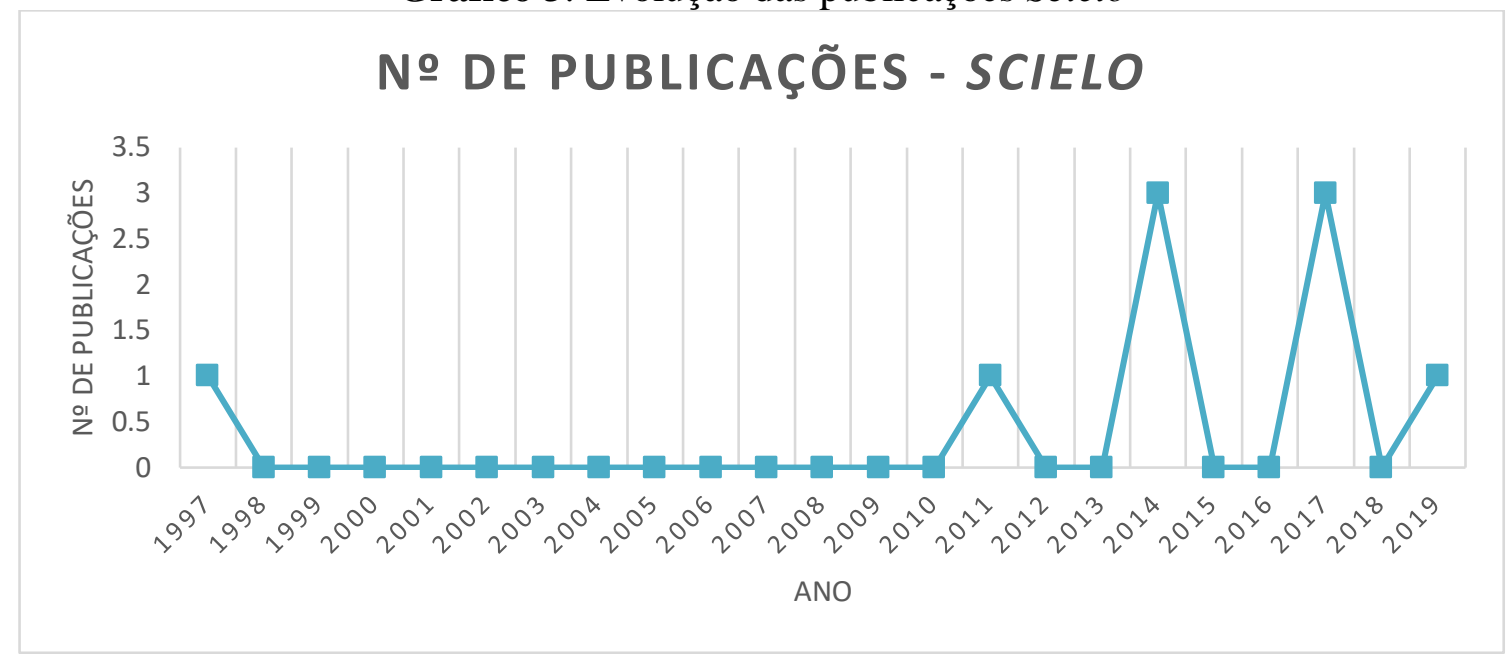

Fonte: Autores.

Pode-se observar que as publicações acerca do tema começaram a evoluir a partir dos anos 2000 tanto na WOS como na Scopus, a Scielo não apresentou nitidamente essa evolução devido a quantidade reduzida de artigos publicados sobre o assunto do que as duas primeiras.

O ano de 2020 apresentou uma queda nas publicações sobre o tema, mas essa verificação se justifica pelo falo da pesquisa ter sido realizada no dia 29/06/2020. Mas este ano não foi excluído no espaço temporal total da pesquisa, mantendo o escopo da pesquisa os trabalhos relevantes publicados no ano atual. Logo, os estudos sobre logística de distribuição tem apresentado um interesse por parte de pesquisadores.

Considerando o grande número de artigos a serem analisados e partindo para a seleção dos artigos publicados nos últimos 10 anos de 2011 a 2020, a Tabela 4 com a quantidade de artigos por base de dados foi elaborada.

Tabela 4. Número de artigos publicados nos últimos 10 anos de acordo com a base de dados

\begin{tabular}{l|l|l}
\hline Artigos -2011 a 2020 & Inglês & Português \\
\hline Web of Science & 274 & 4 \\
\hline Scopus & 3361 & 15 \\
\hline Scielo & 4 & 4 \\
\hline
\end{tabular}

Fonte: Autores.

Quando se inicia a análise das citações, utilizando a metodologia de Lacerda, et al., (2012), para cada base de dados os artigos que somavam cerca de $85 \%$ foram selecionados, resultando no número de artigos apresentado na Tabela 5.

Tabela 5. Número de artigos que somam cerca de $85 \%$ das citações

\begin{tabular}{l|l|l}
\hline Cerca de 85\% das citações & Inglês & Português \\
\hline Web of Science & 55 & 3 \\
\hline Scopus & 999 & 2 \\
\hline Scielo & 1 & 1 \\
\hline
\end{tabular}

Fonte: Autores.

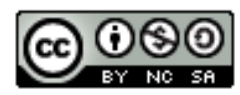


Citação (APA): Bertolo, R. S., \& Freitas, R. R. (2021). Fatores que auxiliam na localização de Centro de Distribuição: Uma Análise Bibliométrica. Brazilian Journal of Production Engineering, 7(1), 129-141.

Ao nível de confiabilidade de $90 \%$, obteve-se para a base WOS 30 artigos e para a base Scopus um total de 63 artigos. A partir da leitura de títulos e resumo obteve-se o seguinte portfólio de artigos para a realização da análise qualitativa (Tabela 6):

Tabela 6. Portfólio final de artigos

\begin{tabular}{l|l|l}
\hline Portfólio Final & Inglês & Português \\
\hline Web of Science & 6 & 1 \\
\hline Scopus & 5 & 0 \\
\hline Scielo & 0 & 0 \\
\hline
\end{tabular}

Fonte: Autores.

Assim, foram selecionados os 11 artigos finais, como mostra a Tabela 7. Observou-se que 1 artigo estava repetido em duas bases de dados, e mediante leitura de títulos e resumos nenhum artigo da base Scielo foi selecionado e da base Scopus não foram selecionados artigos para a busca das tags em português.

Tabela 7. Portfólio final com o título dos artigos

\begin{tabular}{|c|c|c|c|c|}
\hline Item & Título & Autores & Ano & Base \\
\hline 1 & $\begin{array}{l}\text { A multi-criteria decision making approach for } \\
\text { location planning for urban distribution } \\
\text { centers under uncertainty }\end{array}$ & $\begin{array}{l}\text { Awasthi, A., Chauhan, } \\
\text { S. S., \& Goyal, S. K. }\end{array}$ & 2011 & $\begin{array}{l}\text { WOS e Scopus } \\
\text { - Inglês }\end{array}$ \\
\hline 2 & $\begin{array}{l}\text { Optimal location selection for an } \\
\text { international distribution center by using a } \\
\text { new hybrid method }\end{array}$ & Kuo, M. & 2011 & WOS - Inglês \\
\hline 3 & $\begin{array}{l}\text { Incorporating location, inventory and price } \\
\text { decisions into a supply chain distribution } \\
\text { network design problem }\end{array}$ & $\begin{array}{l}\text { Ahmadi-Javid, A., \& } \\
\text { Hoseinpour, P. }\end{array}$ & 2015 & WOS - Inglês \\
\hline 4 & $\begin{array}{l}\text { A study of distribution center location based } \\
\text { on the rough sets and interactive multi- } \\
\text { objective fuzzy decision theory }\end{array}$ & $\begin{array}{l}\text { Liu, S., Chan, F. T. S., } \\
\quad \& \text { Chung, S. H. }\end{array}$ & 2011 & WOS - Inglês \\
\hline 5 & $\begin{array}{l}\text { Location optimization of multiple distribution } \\
\text { centers under fuzzy environment }\end{array}$ & $\begin{array}{l}\text { Wang, Y., Ma, X., } \\
\text { Wang, Y., Mao, H., \& } \\
\text { Zhang, Y. }\end{array}$ & 2012 & WOS - Inglês \\
\hline 6 & $\begin{array}{l}\text { A multi-objective chance-constrained } \\
\text { network optimal model with random fuzzy } \\
\text { coefficients and its application to logistics } \\
\text { distribution center location problem }\end{array}$ & $\begin{array}{l}\text { Xu, J., Yao, L., \& } \\
\text { Zhao, X. }\end{array}$ & 2011 & WOS - Inglês \\
\hline 7 & $\begin{array}{c}\text { Análise e localização de centros de } \\
\text { armazenamento e triagem de resíduos sólidos } \\
\text { urbanos para a rede de logística reversa: um } \\
\text { estudo de caso no município de São Mateus, } \\
\text { ES }\end{array}$ & $\begin{array}{l}\text { Ferri, G. L., Chaves, } \\
\text { G. L. D., \& Ribeiro, G. } \\
\text { M. }\end{array}$ & 2014 & $\begin{array}{l}\text { WOS - } \\
\text { Português }\end{array}$ \\
\hline 8 & $\begin{array}{l}\text { Comparative analysis of multi-criteria } \\
\text { decision making methodologies and } \\
\text { implementation of a warehouse location } \\
\text { selection problem }\end{array}$ & $\begin{array}{l}\text { Ozcan, T., Çelebi, N., } \\
\text { \& Esnaf, S. }\end{array}$ & 2011 & Scopus - Inglês \\
\hline 9 & $\begin{array}{l}\text { Location and allocation decisions for multi- } \\
\text { echelon supply chain network - A multi- } \\
\text { objective evolutionary approach }\end{array}$ & $\begin{array}{l}\text { Shankar, B. L., } \\
\text { Basavarajappa, S., } \\
\text { Chen, J. C. H., \& } \\
\text { Kadadevaramath, R. S. }\end{array}$ & 2013 & Scopus - Inglês \\
\hline 10 & $\begin{array}{c}\text { Location selection of city logistics centers } \\
\text { under sustainability }\end{array}$ & $\begin{array}{l}\text { Rao, C., Goh, M., } \\
\text { Zhao, Y., \& Zheng, J. }\end{array}$ & 2015 & Scopus - Inglês \\
\hline 11 & $\begin{array}{l}\text { A hybrid multi-objective approach to } \\
\text { capacitated facility location with flexible } \\
\text { store allocation for green logistics modeling }\end{array}$ & $\begin{array}{l}\text { Harris, I., Mumford, C. } \\
\text { L., \& Naim, M. M. }\end{array}$ & 2014 & Scopus - Inglês \\
\hline
\end{tabular}

Fonte: Autores. 
Com isso, torna-se possível iniciar a análise qualitativa e discussão dos artigos selecionados.

Awasthi et al., (2011) utilizaram uma abordagem de tomada de decisão de múltiplos critérios (Fuzzy) para planejar a localização de centros de distribuição urbanos sob incertezas. Segundo os autores os critérios para avaliar possíveis locais para instalar centros urbanos são acessibilidade, segurança, conectividade com transporte multimodal, custos, impacto ambiental, disponibilidade de recursos, conformidade com as regularizações de frete sustentável, possibilidade de expansão e qualidade de serviço.

Kuo (2011), em seu estudo, buscou selecionar o local ideal para um centro de distribuição internacional, usando um modelo híbrido, combinando Fuzzy e AHP. Os critérios foram agrupados, como mostra a Tabela 8:

Tabela 8. Critérios utilizados para selecionar um local para um CD

\begin{tabular}{l|l}
\hline Conveniência & - Extensão de conveniência de transporte \\
& - Serviço único \\
& - Habilidades de informação \\
\hline Custo & - Taxa do Porto \\
& - Tempo de transbordo \\
& - Instalações do porto e armazém \\
\hline Condições do porto & - Resistência de localização \\
& - Sistema de operação portuária \\
\hline Capacidade operacional & - Volume de importação / exportação \\
& - Densidade de companhias marítimas \\
\hline
\end{tabular}

Fonte: Autores.

A Tabela acima considera as questões portuárias, através de conveniência, custo, condições de porto e a capacidade operacional dos portos para selecionar um local para um CD. Kuo (2011), também analisa critérios simultaneamente existentes como independentes e dependentes, e o método híbrido pode lidar com um. Este estudo encontra uma melhor localização no Porto de Cingapura (Cingapura). E com isso, o método de avaliação feito pelo autor pode obter a falta entre o ideal alternativa e cada alternativa, e a ordem de classificação das alternativas podem ser facilmente encontradas.

Liu et al. (2011), fizeram o estudo da localização de centro de distribuição, propondo um novo algoritmo heurístico híbrido, combinando métodos de conjuntos brutos e a lógica Fuzzy. Para isso, foi feita uma separação de fatores quantitativos e qualitativos. Os fatores quantitativos envolveram: custos de construção, custos de recursos, custos de transporte e custos operacionais. Os fatores qualitativos envolveram ambiente de negócios, características do produto, oferta de trabalho, serviços, ambiente de competição, ambiente político e outras condições.

Wang et al. (2012), apresentou um estudo de otimização da localização de vários centros de distribuição em ambiente difuso, e também foi utilizado a teoria Fuzzy. Os critérios apontados foram: ambiente natural, transporte, ambiente de negócios, condição de abastecimento e impacto ambiental.

Ahmadi-Javid e Hoseinpour (2015) estudaram o problema de localização, estoque e maximização de lucro de uma rede de distribuição de cadeia de suprimentos de múltiplos

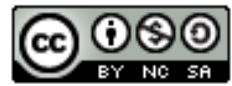


produtos com demandas sensíveis ao preço, ou seja, vários centros de distribuição. E também para dois casos: centros de distribuição não capacitados e centros de distribuição capacitados.

Shankar et al. (2013) apresentaram um artigo de decisões de localização e alocação para rede de cadeia de suprimentos de múltiplos escalões, considerando número de fornecedores e suas capacidades para cada matéria prima, número de plantas potenciais, CDs e suas capacidades, custo de fabricação de cada matéria-prima em cada fornecedor e custo de transporte, custo de produção e estoque em cada planta, custos de distribuição por produto por par de planta - CD, custo de produção do produto em cada CD e custo de transporte por par de CD - CZ (zonas de clientes), número de CZs e suas demandas e taxa mínima de atendimento (fração da demanda atendida) a ser mantido.

Xu et al. (2011) apresentaram um ótimo modelo de rede restrita para aplicação ao problema de localização de centro de distribuição logística, também foi utilizado o coeficiente Fuzzy. Neste estudo foram considerados:

(1) O número de clientes e fornecedores é conhecido.

(2) O número de CDs potenciais e suas capacidades máximas são conhecidos.

(3) Os clientes recebem produtos de um único $C D$.

(4) A quantidade demandada pelos produtos e os custos de transporte são considerados como variáveis fuzzy aleatórias.

(5) Uma vez que o gerente desta empresa deseja reduzir custos e encontrar um modelo ótimo de estratégia de rede, o estoque foi considerado zero.

Ozcan et al. (2011) fez uma análise comparativa entre as metodologias de tomada de decisão multicritério (AHP, TOPSIS, ELECTRE e a Teoria de Gray) para seleção de localização de armazéns, e os critérios considerados foram: preço unitário $\left(\$ / \mathrm{m}^{2}\right)$, capacidade de retenção de estoque (unidade), distância média para lojas $(\mathrm{km})$, distância média aos principais fornecedores $(\mathrm{km})$ e flexibilidade de movimento. TOPSIS e ELECTRE foi a melhor alternativa para o método devido à diferença referências em fórmulas usadas na fase de padronização de desempenho critérios de alternativas contra critérios.

Ferri et al. (2014) não trouxe um estudo precisamente de centros de distribuição, mas sim de centro de armazenagem e triagem de resíduos sólidos urbanos. Este artigo apresenta uma metodologia baseada em um modelo matemático de localização de centros de armazenamento e triagem de RSU para municípios que permite centralizar os resíduos em uma espécie de centro de distribuição reverso, para agrupar os RSU coletados. Os critérios utilizados foram: Locais geradores de RSU, locais candidatos e custo unitário de transporte; Estimativa de RSU gerado; Faixas de capacidade; Custo fixo de instalação e de operação.

Rao et al. (2015) trata de uma seleção da localização dos centros de logística da cidade (CLC) sob sustentabilidade, tendo como critérios dimensões econômicas, ambientais e sociais. Para mostrar sua eficácia e praticidade do método destacou um novo híbrido de 2 tuplas pedido operador de média ponderada (THOWA).

Harris et al. (2014) traz uma abordagem híbrida para localização de instalação capacitada com alocação flexível de loja para modelagem de logística verde, considerando custos e emissão de 
$\mathrm{CO}_{2}$. Para lidar com custos e emissões de $\mathrm{CO}_{2}$ de alocação utiliza-se modelos de Relaxamento Lagrangiano.

\section{CONCluSÃo}

A análise bibliométrica está sendo muito estudada no ambiente acadêmico, e o estudo realizado com este artigo identificou os fatores que auxiliam na localização de um CD. As bases de dados utilizadas Web of Science - WOS, Scopus e Scielo, o que proporcionou o mapeamento da atividade científica com base no tema, utilizando a princípio todo o período de abrangência dessas três prinicipais bases de dados disponíveis.

Com a definição das bases de dados e determinação das tags, foi realizada a seleção dos materiais para a composição do portfólio de publicações. Seguindo as seguintes etapas de exclusão em cada umas das bases: documentos do tipo artigo em cada uma das plataformas; artigos publicados nos últimos 10 anos; número de corte para os artigos que foram mais citados, conforme o método de Lacerda et al. (2012); para as bases de dados WOS e Scopus ainda definiu-se o número amostral considerado válido para análise; e a leitura dos títulos e resumos dos artigos das 3 bases de dados nos 2 idiomas. Sendo realizada ainda, a análise qualitativa do portfólio.

A primeira pesquisa feita, sem filtros, mostrou um maior número de artigos sobre o tema na base de dados Scopus, mas com todas as etapas de exclusão aplicados a base de dados Scielo não apresentou nenhum artigo para a composição do portfólio. A partir das palavras-chave iniciais, foram identificados 7.628 artigos juntando todas as bases pesquisas e após os filtros aplicados foram 11 artigos selecionados para a discussão sobre o tema.

A maioria dos trabalhos trabalhou com a teoria Fuzzy e outros métodos de análise multicritérios. E pode-se observar que acesso, custo, impacto ambiental e recursos são mencionados em vários artigos do portfólio final analisado.

Dessa forma, o objeto deste estudo foi atingido e recomenda-se para trabalhos futuros o estudo outras bases de dados diferentes para uma comparação com as bases estudadas.

\section{REFERÊNCIAS}

Ahmadi-Javid, A., \& Hoseinpour, P. (2015). Incorporating location, inventory and price decisions into a supply chain distribution network design problem. Computers \& Operations Research, 56, 110-119.

Awasthi, A., Chauhan, S. S., \& Goyal, S. K. (2011). A multi-criteria decision-making approach for location planning for urban distribution centers under uncertainty. Mathematical and Computer Modelling, 53(1-2), 98-109.

Ballou, R. H. (1993). Logística empresarial: transportes, administração de materiais e distribuição fiscal. São Paulo: Atlas.

(2001). Gerenciamento da cadeia de suprimentos: planejamento, organização e logística empresarial. Porto Alegre: Bookmann.

Boyle, F., \& Sherman, D. (2006). The productand its development. The Serials Librarian, Philadelphia, 49(3), 147-153.

Carvalho, N. L. A., Ribeiro, P. C. C., Oliveira, L. K., \& Silva, J. E. A. R. (2019). Criteria to implement UDCs in historical cities: a Brazilian case study. European Transport, 72, 1-29. 
Citação (APA): Bertolo, R. S., \& Freitas, R. R. (2021). Fatores que auxiliam na localização de Centro de Distribuição: Uma Análise Bibliométrica. Brazilian Journal of Production Engineering, 7(1), 129-141.

Cay, T., \& Uyan, M. (2013). Evaluation of reallocation criteria in land consolidation studies using the Analytic Hierarchy Process (AHP). Land Use Policy, 30(1), 541- 548.

Chow, G. (1984). Microcomputers in Logistics. Logistics and Transportation Review, 20(4), 471-501.

Chueke, G. V., \& Amatucci, M. (2015). O que é bibliometria? Uma introdução ao Fórum. Internext, 10(2), 1-5.

Cunha, L. P., Angélico, M. G., \& Medeiros Neta, O. M. (2018). Mapeamento Bibliométrico Da Produção Científica: Elaborando Conhecimento Sobre Pestalozzi. Revista Brasileira de Iniciação Científica, Itapetininga, 5(4), 58-69.

Dupont, W. D., \& Plummer, W. D. (1990). Power and sample size calculations: a review and computer program. Controlled clinical trials, 11(2), 116-128.

E-commerce Brasil. (2019). Estudo aponta tendências de logística e transporte do país em 2020. Recuperado de https://www.ecommercebrasil.com.br/noticias/tendencias-logistica-transporte$\underline{2020 /}$

Ferri, G. L., Chaves, G. L. D., \& Ribeiro, G. M. (2014). Análise e localização de centros de armazenamento e triagem de resíduos sólidos urbanos para a rede de logística reversa: um estudo de caso no município de São Mateus, ES. Production, 25(1).

Gomes, V. A. P., \& Freitas, R. R. (2019). Wind Farm Implementation Factors: A Bibliometric Analysis. International Journal of Advanced Engineering Research and Science, 6, 58-64.

Harper, R. L. (2010). Warehouse Technology in the Supply Chain Management Systems. Annual Reliability and Maintainability Symposium (RAMS). Florida University.

Harris, I., Mumford, C. L., \& Naim, M. M. (2014). A hybrid multi-objective approach to capacitated facility location with flexible store allocation for green logistics modeling. Transportation Research Part E: Logistics and Transportation Review, 66, 1-22.

ILOS. Especialistas em Logítica e Supply Chain. (2014). Consulta geral a homepage oficial. Recuperado de http://www.ilos.com.br

Custos Logístico no Brasil. (2017). Portal Ilos. Recuperado de http://www.ilos.com.br/web/analise-de-mercado/relatorios-de-pesquisa/custos-logisticos-nobrasil/

Kuo, M. (2011). Optimal location selection for an international distribution center by using a new hybrid method. Expert Systems with Applications, 38(6), 7208-7221.

Lacerda, R. T. O., Ensslin, L., \& Ensslin, S. R. (2012). Uma análise bibliométrica da literatura sobre estratégia e avaliação de desempenho. Gestão \& Produção, 19(1).

Liu, S., Chan, F. T. S., \& Chung, S. H. (2011). A study of distribution center location based on the rough sets and interactive multi-objective fuzzy decision theory. Robotics and ComputerIntegrated Manufacturing, 27(2), 426-433.

Morales, S. R., Morabito, R., \& Widmer, J. A. (1997). Otimização do carregamento de produtos paletizados em caminhões. Gestão \& Produção, 4(2), 234-252.

Noronha, D. P., Kiyotani, N. M., \& Juanes, I. A. S. (2002). Produção científica de docentes na área de comunicação. Encontro nacional de centros de informação da área de comunicação, Salvador, Anais Salvador.

Ozcan, T., Çelebi, N., \& Esnaf, S. (2011). Comparative analysis of multi-criteria decision making methodologies and implementation of a warehouse location selection problem. Expert Systems with Applications, 38(8), 9773-9779. 
Pelúcio, C. (2020). Impacto do coronavírus no transporte de cargas chega a $26 \%$ segundo dados do Decope. NTC\&Logística. Recuperado de https://www.portalntc.org.br/noticias/rodoviario/6074-impacto-do-coronavirus-no-transportede-cargas-chega-a-26-segundo-dados-do-decope.html

Ramos, P. T. R. (2015). Estudo para implantação de Centro de Distribuição de produtos farmacêuticos na cidade de Uberlândia (MG). 2015. Dissertação (Mestrado) - Faculdade de Engenharia Civil, Universidade Federal de Uberlândia, Uberlândia.

Rao, C., Goh, M., Zhao, Y., \& Zheng, J. (2015). Location selection of city logistics centers under sustainability. Transportation Research Part D: Transport and Environment, 36, 29-44.

Samor, G. (2018). Brasil desperdiça $R \$ 100$ bi em logística. Jornal de Minas Gerais. Disponível em:

$<$ https://www.em.com.br/app/noticia/economia/2018/06/05/internas_economia,964437/brasildesperdica-r100-bi-em-logistica.shtml>. Acesso em: 29 jun. 2020.

Shankar, B. L., Basavarajappa, S., Chen, J. C. H., \& Kadadevaramath, R. S. (2013). Location and allocation decisions for multi-echelon supply chain network - A multi-objective evolutionary approach. Expert Systems with Applications, 40(2), 551-562.

Shannon, R. E., \& Ignizio, J. P. (1970). A heuristic programming algorithm for warehouse location. AIIE Transactions, 2(4), 334-339.

Wang, Y., Ma, X., Wang, Y., Mao, H., \& Zhang, Y. (2012). Location optimization of multiple distribution centers under fuzzy environment. Journal of Zhejiang University SCIENCE A, 13, 782-798.

Xu, J., Yao, L., \& Zhao, X. (2011). A multi-objective chance-constrained network optimal model with random fuzzy coefficients and its application to logistics distribution center location problem. Fuzzy Optimization and Decision Making, 10, 255-285. 